\title{
Juvenile Scleroderma: A Referral Center Experience
}

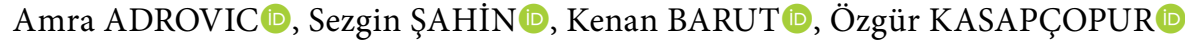 \\ Department of Pediatric Rheumatology, İstanbul University, Cerrahpaşa Faculty of Medicine, İstanbul, Turkey
}

\begin{abstract}
Objectives: This study aims to evaluate the demographic and clinical features, laboratory data, treatment modalities, and outcomes of juvenile systemic sclerosis (JSS) and juvenile localized scleroderma (JLS) patients at a referral pediatric rheumatology center in Turkey.

Patients and methods: Medical records of a total of 57 patients, including 29 with JSS (1 male, 28 females; mean age $18.3 \pm 3.2$ years; range 14 to 27 years) and 28 with JLS ( 6 males, 22 females; mean age 14.4 4.8 years; range 6 to 23 years), diagnosed betweenJanuary 2006 and Mart 2015 and followed-up for at least six months were evaluated in this retrospective longitudinal study. All medical records were retrospectively analyzed for demographic, clinical, and laboratory findings.

Results: Mean age at disease onset was $9.9 \pm 4.2$ years and $7.7 \pm 3.9$ years for JSS and JLS, respectively. Mean ages at diagnosis and at the time of study were lower in JLS: $9.1 \pm 3.5$ years vs. $11.7 \pm 3.7$ years and $14.4 \pm 4.8$ years vs. $18.3 \pm 3.2$ years, respectively. Mean disease duration was $7.8 \pm 5.2$ years and $8.0 \pm 4.3$ years for JSS and JLS, respectively. Among JSS patients, interstitial lung disease was seen in eight (27\%), pulmonary hypertension in three $(10 \%)$, and arrhythmia in one (3\%). One JSS patient (3\%) died as a consequence of cardiac sclerosis. Corticosteroids with methotrexate were used in 29 JSS patients (100\%) and in 21 JLS patients (75\%). Patients with vasculopathy were treated with nifedipine $(n=18,62 \%)$ and bosentan $(n=12,41 \%)$. Internal organ involvement was treated with high-dose cyclophosphamide $(n=10,34 \%)$ or biological agent $(n=3,10 \%)$.

Conclusion: Close monitoring of internal organ involvement is of great importance in preventing disease-related complications in JSS and JLS. Although rare, vital organ involvement has a devastating effect on prognosis. Biological agents represent an option for patients resistant to standard immunosuppressive treatment.

Keywords: Juvenile localized scleroderma; juvenile systemic sclerosis; Raynaud's phenomenon; skin stiffening.
\end{abstract}

Juvenile scleroderma (JS) is a rarely seen chronic connective tissue disorder characterized by stiffening of the skin. Storage of collagen in the skin, subcutaneous tissue, and internal organs is the basic pathophysiological mechanism of the disease. According to organ involvement, the disease is divided into two main forms as systemic and localized scleroderma. ${ }^{1}$

Juvenile localized scleroderma (JLS) is more frequent in childhood. Although internal organ involvement is rare, approximately onefourth of patients with JLS are shown to have musculoskeletal complaints. The disease is considered to be benign but it may cause significant functional and cosmetic deformity. ${ }^{2,3,5,7,8}$ Studies on localized scleroderma among adults and children generally report marked diagnostic delay and present a diverse range of treatment modalities. ${ }^{7,8}$

Juvenile systemic sclerosis (JSS) is a quite rare condition characterized with multisystemic involvement and poorer prognosis. ${ }^{1-4}$ Many data regarding disease features and treatment options in JS are based on studies among adults. Studies among children are insufficient and most are conducted in a retrospective manner or in

Received: July 13, 2017 Accepted: December 13, 2017 Published online: January 18, 2018

Correspondence: Özgür Kasapçopur, MD. İstanbul Üniversitesi Cerrahpaşa Tıp Fakültesi Çocuk Romatolojisi Bilim Dalı, 34096 Fatih, İstanbul, Turkey. Tel: +90 212 - 4143201 e-mail: ozgurkasapcopur@hotmail.com 
comparison with adults, with the exception of an ongoing multicentric, multinational study among children with systemic sclerosis. ${ }^{6,9-13}$ In general, there is a lack of standard protocol on assessment and treatment modality of JS patients with an indispensable need for prospective investigation in this area.

As far as we know, this is the largest single center JS cohort including both systemic and localized form of the disease. In this study, we aimed to evaluate the demographic and clinical features, laboratory data, treatment modalities, and outcomes of JSS and JLS patients at a referral pediatric rheumatology center in Turkey.

\section{PATIENTS AND METHODS}

Medical records of a total of 57 patients, including 29 with JSS (1 male, 28 females; mean age $18.3 \pm 3.2$ years; range 14 to 27 years) and 28 with JLS (6 males, 22 females; mean age 14.4 \pm 4.8 years; range 6 to 23 years), diagnosed between January 2006 and Mart 2015 at the İstanbul University, Cerrahpaşa Faculty of Medicine and followed-up for at least six months were evaluated in this retrospective longitudinal study. One patient with mixed connective tissue disorder was excluded. The study protocol was approved by the Institutional Review Board at the İstanbul University, Cerrahpaşa Faculty of Medicine. The study was conducted in accordance with the principles of the Declaration of Helsinki.

All patients in systemic scleroderma group met the Pediatric Rheumatology European Society/American College of Rheumatology/ European League against Rheumatism provisional classification criteria for JSS. ${ }^{4}$ Diagnosis of localized scleroderma was established by the same experienced pediatric rheumatologist.

All medical records were retrospectively analyzed for demographic, clinical, and laboratory findings. Data recorded for each patient included age of the patient at the time of study, age at disease onset, age at diagnosis, sex, disease subtype, and disease, follow-up, and treatment durations.

Organ involvement at the time of first manifestation and anytime during the follow-up was recorded. Skin involvement was determined by the skin induration, edema, or sclerodactyly proximal or distal to metacarpophalangeal joints. Vascular system involvement was defined as presence of Raynaud's phenomenon (RP), digital infarcts, digital scars, or abnormal nailfold capillaries and capillaroscopy findings (for patients with available data). Due to lack of consensus on interpretation of nailfold capillaroscopy findings in pediatric population, many centers do not routinely use this procedure for the evaluation of patients. We divided the nailfold capillaroscopy findings in three main groups as early, active, and late scleroderma pattern. ${ }^{14}$ Lung involvement was defined by abnormal chest radiography or high-resolution computed tomography findings, reduced does carbon monoxide diffusing capacity, or reduced forced vital capacity. Cardiac disease was diagnosed by the presence of arrhythmias, heart failure, or pulmonary hypertension $(\mathrm{PH})$ registered during the annual echocardiography. Patients with high pulmonary artery pressure on echocardiography underwent angiography for the definitive diagnosis of $\mathrm{PH}$. Musculoskeletal involvement was defined by the presence of muscle weakness, arthritis, arthralgia, or tendon friction rubs. Gastrointestinal tract involvement was determined by the presence of dysphagia, gastroesophageal reflux, diarrhea, or weight loss. Raised creatinine levels, proteinuria, renal crisis, or persistent arterial hypertension defined renal disease. History of seizures, peripheral neuropathy, or abnormal findings on brain magnetic resonance imaging (MRI) was accepted as central nervous system involvement.

Hemogram, acute phase markers, kidney and liver function tests, urine analysis, serum levels of autoantibodies, including antinuclear antibodies, and anti-topoisomerase I were recorded at the time of diagnosis and during the follow-up. Abnormal values were determined by using the normal range of our laboratory standards.

The way of measuring the disease activity is still a matter of controversy. Skin stiffness of patients with JSS was assessed by using the modified Rodnan skin score (mRSS) ${ }^{15}$ and the disease severity according to organ involvement was measured by using the disease severity score. ${ }^{16}$ Skin activity and skin damage in patients with JLS were assessed by Localized Scleroderma Cutaneous Assessment 
Table 1. Demographic features of patients with juvenile scleroderma

\begin{tabular}{|c|c|c|c|c|c|c|c|}
\hline & \multicolumn{3}{|c|}{ Juvenile systemic sclerosis $(n=29)$} & \multicolumn{3}{|c|}{ Juvenile localized scleroderma $(n=28)$} & \multirow[b]{2}{*}{$p$} \\
\hline & $\mathrm{n}$ & $\%$ & Mean \pm SD & $\mathrm{n}$ & $\%$ & Mean \pm SD & \\
\hline Gender & & & & & & & 0.191 \\
\hline Female & 28 & 97 & & 22 & 79 & & \\
\hline Male & 1 & 3 & & 6 & 21 & & \\
\hline Age at the disease onset & & & $9.9 \pm 4.2$ & & & $7.7 \pm 3.9$ & 0.048 \\
\hline Age at diagnosis & & & $11.7 \pm 3.7$ & & & $9.1 \pm 3.5$ & 0.009 \\
\hline Age at the time of study & & & $18.3 \pm 3.2$ & & & $14.4 \pm 4.8$ & 0.005 \\
\hline Mean disease duration & & & $7.8 \pm 5.2$ & & & $8.0 \pm 4.3$ & 0.581 \\
\hline Mean follow-up duration & & & $6.5 \pm 4.5$ & & & $5.3 \pm 4.6$ & 0.157 \\
\hline Mean duration of treatment & & & $5.2 \pm 3.7$ & & & $4.5 \pm 4.1$ & 0.32 \\
\hline
\end{tabular}

Tool (LoSCAT) score, which consists of Localized Scleroderma Skin Damage Index (LoSDI) and Skin Severity Index (LoSSI). ${ }^{7.17}$ Data on used treatment were assessed cumulatively, from the beginning of treatment until the time of study.

\section{Statistical analysis}

Statistical analysis was performed by using the IBM SPSS Statistics for Windows 21.0 software (Released 2012, IBM Corp., Armonk, NY, USA). Distributional properties of continuous variables were expressed in mean \pm standard deviation and median (minimum-maximum). Categorical variables were presented with frequency and percentage (\%). The Shapiro-Wilk test was used to examine the differences in the distributions of continuous variables. The Mann-Whitney U and the Chi-square tests were used to determine differences between continuous and categorical variables of two groups. Statistical significance level was obtained at a $p$ value of $\leq 0.05$.

\section{RESULTS}

Mean ages at diagnosis and at the time of study were significantly lower in patients with JLS, compared to JSS $(9.1 \pm 3.5$ years vs. $11.7 \pm 3.7$ years and $14.4 \pm 4.8$ years vs. $18.3 \pm 3.2$ years, respectively). There was no significant difference in other demographic features (Table 1). Clinical findings of patients were shown in Table 2. RP was significantly more frequent among JSS patients at disease onset: 27 (93\%) vs. four (14\%). At the end of follow-up, 17 JSS patients (58\%) continued to have RP. Digital ulceration was seen only in JSS patients at disease onset and at last examination ( $n=24,83 \%$ and $n=13,44.8 \%$, respectively).

Findings of nailfold capillaroscopy were obtained in 27 JSS patients: 16 patients (60\%) had early, 10 patients (37\%) active, and one patients (3\%) had late scleroderma pattern. Interstitial lung disease was present in six (20\%) and eight (27\%) JSS patients at the disease onset and on the last

Table 2. Cumulative data on main clinical features and serological profile of juvenile scleroderma patients

\begin{tabular}{|c|c|c|c|c|c|}
\hline & \multicolumn{2}{|c|}{ Juvenile systemic sclerosis $(n=29)$} & \multicolumn{2}{|c|}{ Juvenile localized scleroderma $(n=28)$} & \multirow[b]{2}{*}{$p$} \\
\hline & $\mathrm{n}$ & $\%$ & $\mathrm{n}$ & $\%$ & \\
\hline Raynaud's phenomenon & 27 & 93 & 4 & 14 & $<0.001$ \\
\hline Digital ulceration & 24 & 83 & 0 & 0 & $<0.001$ \\
\hline Interstitial lung disease & 8 & 27 & 0 & 0 & 0.005 \\
\hline Pulmonary hypertension & 3 & 10 & 0 & 0 & 0.492 \\
\hline Arrhythmia & 1 & 3 & 0 & 0 & 1.000 \\
\hline Arthralgia & 22 & 76 & 6 & 24 & 0.012 \\
\hline Arthritis & 23 & 81 & 5 & 19 & 0.065 \\
\hline Calcinosis & 2 & 7 & 0 & 0 & 0.462 \\
\hline Dysphagia & 8 & 27 & 0 & 0 & 0.011 \\
\hline Gastroesophageal reflux & 8 & 27 & 0 & 0 & 0.002 \\
\hline \multicolumn{6}{|l|}{ Serological profile } \\
\hline Anti-nuclear antibodies & 29 & 100 & 21 & 75 & 0.004 \\
\hline Anti-topoisomerase I antibody & 6 & 20 & 0 & 0 & 0.011 \\
\hline
\end{tabular}


Table 3. Treatment modalities in patients with juvenile systemic sclerosis and juvenile localized scleroderma

\begin{tabular}{|c|c|c|c|c|c|c|c|}
\hline & \multicolumn{3}{|c|}{ Juvenile systemic sclerosis $(n=29)$} & \multicolumn{3}{|c|}{ Juvenile localized scleroderma $(n=28)$} & \multirow[b]{2}{*}{$p$} \\
\hline & $\mathrm{n}$ & $\%$ & Mean \pm SD & $\mathrm{n}$ & $\%$ & Mean \pm SD & \\
\hline \multicolumn{8}{|c|}{ Frequency of used medications } \\
\hline Corticosteroids & 29 & 100 & & 16 & 57 & & $<0.001$ \\
\hline Methotrexate & 29 & 100 & & 21 & 75 & & 0.045 \\
\hline Mycophenolate-mofetil & 7 & 24 & & 0 & 0 & & 0.01 \\
\hline Cyclophosphamide & 10 & 34 & & 0 & 0 & & 0.001 \\
\hline Biological agent & 3 & 10 & & 0 & 0 & & 0.237 \\
\hline Nifedipine & 18 & 62 & & 0 & 0 & & $<0.001$ \\
\hline Bosentan & 12 & 41 & & 0 & 0 & & $<0.001$ \\
\hline \multicolumn{8}{|l|}{ Duration of treatments } \\
\hline Corticosteroids & & & $51 \pm 40.8$ & & & $15.3 \pm 17.9$ & $<0.001$ \\
\hline Methotrexate & & & $59.3 \pm 39.9$ & & & $57.0 \pm 52.7$ & 0.39 \\
\hline Mycophenolate-mofetil & & & $16.3 \pm 12.8$ & & & NA & NA \\
\hline
\end{tabular}

clinical examination, respectively. $\mathrm{PH}$ was seen in three JSS patients (10\%) while one JSS patient (3\%) had arrhythmia (consistent with WolffParkinson-White pattern) at the disease onset. Two (7\%) of previously mentioned three patients had $\mathrm{PH}$ at the time of study. Two patients from JSS group (7\%) developed calcinosis during the follow-up. Disease duration to calcinosis development was five years. There were no patients with neither neurological nor renal involvement at the disease onset, albeit one patient (3\%) from JSS group who developed neurological involvement. Disease duration to neurological involvement of the patient was five years.

At disease onset, only one patient from JSS group (3\%) had elevated sedimentation rate and $\mathrm{C}$-reactive protein. Acute phase markers in all JLS patients were in normal range. Serological profile of patients was shown in Table 2 .

Data on treatment modality were shown in Table 3. Most patients received corticosteroids but the mean duration of corticosteroids treatment was significantly longer in JSS patients (51 months vs. 15.27 months) $(p<0.001)$. In a high number of patients, glucocorticoids were initially combined with methotrexate (MTX) (standard dose of $25 \mathrm{mg} / \mathrm{m}^{2} /$ week): in 29 (100\%) of JSS and in $21(75 \%)$ of JLS patients. In seven JSS patients (24\%), MTX was replaced by mycophenolate-mofetil (MMF) due to intolerance and poor compliance during the follow-up. Patients with symptoms of vasculopathy (RP, digital ulcerations) were treated with nifedipine (30 mg/day) and/or bosentan (2 mg/kg/day):
18 JSS patients $(62 \%)$ treated with nifedipine and 12 of them, who were unresponsive to nifedipine, were treated with bosentan, at the same time. Among 12 patients treated with bosentan, three were diagnosed with $\mathrm{PH}$. Eight patients (25\%) with interstitial lung disease and two (6.9\%) with gastro-intestinal involvement and high mRSS $(>20)$ were additionally treated with high dose of cyclophosphamide (CYC). Patients with internal organ involvement unresponsive to steroids and disease modifying antirheumatic drugs (DMARDs) were treated with biological agent. Two patients (both with $\mathrm{PH}$ and one with co-exsisting cardiac fibrosis) were treated with rituximab $\left(375 \mathrm{mg} / \mathrm{m}^{2} /\right.$ week, for four consecutive doses). One patient with interstitial lung disease, gastrointestinal involvement, and high mRSS was treated with tocilizumab $(12 \mathrm{mg} / \mathrm{kg} /$ dose, once per month); this patient is still under tocilizumab treatment. A mean time to introduction of biological treatment was $3 \pm 1.7$ years.

The mean J4 score did not significantly change during the follow-up: 4 (range, 1-12) at the disease onset and again 4 (range, 0-15) at the time of study. The mean mRSS in JSS patients was 17 (range, 0-40) at the time of the study. Unfortunately, records on mRSS at the disease onset were not available. Mean LoSCAT for JLS patients was $14.5 \pm 8.0$, mean LoSSI was $4.3 \pm 2.8$, and mean LoSDI was $10.3 \pm 6.5$. There was no statistically significant difference between JLS subtypes according to LoSCAT.

Eight JSS patients (27\%) had dysphagia at time of the study. Two JSS patients (7\%) still had high mean pulmonary artery pressure 
(>25 mmHg) at the time of the study. One JSS patient (3\%) had severe headache due to neurological involvement, characterized with brain calcifications and hyperintense white matter signals on the MRI.

The mean duration of follow-up was $6.5 \pm 4.5$ years and $5.3 \pm 4.6$ years for JSS and JLS, respectively. There was no significant change in disease activity score (J4 score for JSS and LoSCAT for JLS) during the follow-up. One JSS patient died due to heart failure as a consequence of cardiac fibrosis. This patient died on the sixth year of the disease. One JSS patient (3\%) developed neurological involvement on the fifth year of the disease.

\section{DISCUSSION}

Juvenile scleroderma is an extremely rare disease with many questions waiting to be answered. Up to date, studies on this topic are scarce, except for an ongoing multicentric inception cohort that may reveal new findings. ${ }^{6}$ Recent developments in treatment options, particularly biological agents have possibly influenced the clinical course and the prognosis of the disease.

We may indicate that JS (both JSS and JLS) is predominantly a disease of the females, since the majority of our patients were girls $(97 \%$ of JSS and 79\% of JLS patients), similar to reports from the literature. $6,9-13,18,21$ Main demographic characteristics of our cohort did not significantly differ from previously reported data.6,9-13,18,21 However, mean ages at diagnosis and at the time of study were significantly lower in patients with JLS, compared to JSS: $9.1 \pm 3.5$ years vs. $11.7 \pm 3.7$ years and $14.4 \pm 4.8$ years vs. $18.3 \pm 3.2$ years, respectively).

Although data from the literature showed that localized form of the disease is more common in childhood, frequency of both disease forms was similar in our cohort. This could be explained by the fact that this is a tertiary medical center with patients from all over the country being referred to our hospital. Additionally, patients with systemic form of the disease undergo obligatory follow-up at pediatric rheumatologic department while some patients with localized scleroderma are referred to dermatologists.
The mean mRSS in our JSS patients was slightly lower compared to results from studies by Russo et al. ${ }^{12}$ and Misra et al. ${ }^{13}$ (17 vs. 22). Unfortunately, we were unable to report any change in skin stiffening during the follow-up since data on mRSS at the disease onset were not available. Furthermore, there is no instrument measuring isolated skin stiffness in JLS. Although the LoSCAT includes skin fibrosis, it also measures other aspects of the disease. This is why assessing clinical changes in routine JS patient care is challenging and includes many subjective variations.

Raynaud's phenomenon is a clinical sign generally first noticed by patient, being present in the $70-80 \%$ of patients. ${ }^{1-3,6,9-13}$ In our cohort, the frequency of RP at the disease onset was even higher (93\%) but showed significant decrease during the follow-up (58\% of JSS patients, $\mathrm{p}<0.05$ ). This result is not surprising since all patients with vasculopathy (e.g. RP, digital ulcerations) were under treatment with vasoactive agents.

Vital organ involvement (lung, cardiovascular system) is rare in childhood.9,10,20-22 In a study by Borowiec et al., ${ }^{21} 86.7 \%$ of patients with JSS had basal interstitial infiltrations in lung while Martini et al. ${ }^{10}$ reported significantly lower percentage of lung fibrosis (29\%). In another cohort from Southeast Asia, the percentage of interstitial lung disease was reported as 65\%. ${ }^{13}$ In our study, interstitial lung disease was less frequent: in six JSS patients (20\%) at the disease onset and in eight JSS patients (27\%) at the last clinical examination. However, the rise in number of patients with interstitial lung disease during the follow-up despite the immunosuppressive treatment opens a question on efficacy of routinely used therapy.

In a study from Argentina, two of 23 patients (8.7\%) had cardiac involvement. ${ }^{12}$ Another study from Southeastern Asia reported PH in 4\% of JSS patients. ${ }^{13}$ In our study, $\mathrm{PH}$ was seen in three JSS patients (10\%) while one patient (3\%) had arrhythmia at the disease onset. The same patient developed cardiac insufficiency during the follow-up. This patient died due to cardiopulmonary insufficiency as a consequence of cardiac fibrosis six years after the disease onset, despite aggressive immunosuppressive treatment. However, there was a significant diagnostic delay 
of five years that could be blamed for the poor outcome, together with unsatisfactory compliance to treatment. The patient received corticosteroids in combination with MTX, which was replaced by MMF due to intolerance to MTX. Pulse CYC treatment was introduced; however, the patient did not collaborate and only two doses have been given. We planned to start biological agent (rituximab) but the patient deceased before therapy was introduced, unfortunately. A patient in a study from Argentina also died in the sixth year after the disease onset, due to complications of the cardiovascular involvement. ${ }^{12}$

Central nervous system and renal involvement are extremely rare. ${ }^{1,6,9-13}$ In our cohort, only one patient $(3 \%)$ had severe headache and brain MRI revealed abnormalities.

Data on clinical outcome vary among different studies. Martini et al. ${ }^{10}$ reported a mortality rate of $11 \%$ and showed that most deceased patients $(73.3 \%)$ died in the first five years after the diagnosis was established. Foeldvari et al. ${ }^{11}$ reported a favorable outcome with overall fiveyear survival of $95 \%$ in a multicentric survey among 135 JSS patients. They showed increased fatal outcome among patients with cardiovascular and renal involvement. ${ }^{11}$ One patient (3\%) from our cohort died in the sixth year after the disease onset. We can speculate that delayed diagnosis and treatment caused a diffuse organ involvement (including heart and lung) in our patient that led to death. The patient received treatment on the fifth year of the disease for one year, which is significantly shorter compared to the mean treatment duration of our JSS cohort (5.21 years).

Therapy of systemic sclerosis is controversial due to rarity of the disease, lack of consensus, and paucity of evidence regarding clinical course and prognosis. Some DMARDs are widely used; however, no drug has been found to have an unequivocal benefit. ${ }^{23,24-26}$ We initially use oral corticosteroids in combination with DMARDs (MTX, mycophenolate-mofetil, CYC) for both disease forms. The treatment duration depends on disease form, clinical course, and disease activity.

In the case of interstitial lung disease, a high dose of CYC has been added to therapy: high dose (1 $\mathrm{g} / \mathrm{m}^{2}$ intravenous/month) during the consecutive first six months. Previous studies reported usage of CYC in $12.9 \%$ of JSS patients, which is lower compared to our cohort where $34 \%$ of JSS patients received CYC. ${ }^{10,28}$ Although studies about MMF in JSS patients are insufficient, reports from adults with interstitial lung disease showed favorable effects of MMF in patients who were inadequate responders to CYC. ${ }^{24,26}$ Those data bring new promises for patients with lung involvement.

We routinely introduce calcium channel blockers (nifedipine) in doses of $0.25-0.5 \mathrm{mg} / \mathrm{kg} /$ day orally in patients with RP. Endothelin antagonists (bosentan) are added in patients with active fingertip ulcerations, despite the regular treatment with calcium channel blockers. ${ }^{28,29}$ In our JSS cohort, 18 patients $(62 \%)$ were treated with nifedipine while $12(41 \%)$ were additionally treated with bosentan (two of them were diagnosed with $\mathrm{PH}$, as well). The percentage of patients receiving calcium channel blockers in our study $(\mathrm{n}=18,62 \%)$ was similar to data reported by Martini et al. ${ }^{10}$ (54\%).

Biological treatment emerges as a useful treatment option in most severe form of the disease; however, supportive data are lacking. There are few reports about anti-interleukin 6 (tocilizumab) and anti-CD20 (rituximab) treatment among adults. ${ }^{25,30-32}$ In our routine practice, a biological agent is added to therapy for patients with multisystemic involvement, with resistance to previous treatment and disease progression. Two patients (two with $\mathrm{PH}$, one with coexisting cardiac fibrosis) were treated with rituximab. Tocilizumab was introduced in therapy in one patient with gastro-intestinal involvement, interstitial lung disease, and high mRSS. Marked decrease in mRSS and physician/patient global assessment score were registered in both patients treated with rituximab, after six months of treatment (mRSS decreased from 40 to 36 and Visual Analog Score decreased from 60 to 40 in patient one, while mRSS decreased from 19 to 16 and Visual Analog Score decreased from 50 to 30 in patient two). Long-term follow-up will reveal the effects of the biological treatment.

Main limitations of our study are its retrospective nature and the limited number of studied patients, due to extreme rarity of the disease worldwide.

In conclusion, close monitoring of the disease status and internal organ involvement are of 
great importance in preventing the disease-related complications in JSS and JLS. Although rare, vital organ involvement has devastating effect on prognosis. Biological agents represent an option for patients resistant to standard immunosuppressive treatment. Nonetheless, multicentric prospective studies might reveal the answers to many questions regarding this rare condition.

\section{Declaration of conflicting interests}

The authors declared no conflicts of interest with respect to the authorship and/or publication of this article.

\section{Funding}

The authors received no financial support for the research and/or authorship of this article.

\section{REFERENCES}

1. Zulian F. Systemic sclerodermas. In: Petty RE, Laxer RM, Lindsley CB, Wedderburn LR, editors. Textbook of Pediatric Rheumatology. 7th ed. Philadelphia: Elsevier; 2016. p. 384-405.

2. Zulian F, Cuffaro G, Sperotto F. Scleroderma in children: an update. Curr Opin Rheumatol 2013;25:643-50.

3. Foeldvari I. Update on juvenile systemic sclerosis. Curr Rheumatol Rep 2015;17:18.

4. Zulian F, Woo P, Athreya BH, Laxer RM, Medsger TA Jr, Lehman TJ, et al. The Pediatric Rheumatology EuropeanSociety/American College of Rheumatology/ European League against Rheumatism provisional classification criteria for juvenile systemic sclerosis. Arthritis Rheum 2007;57:203-12.

5. Gyorgyi Z, Foeldvari I, Weiser P, Szabó A, Constantin $\mathrm{T}$. Recent advances in systemic scleroderma in childhood. Ann Paediatr Rheum 2014;3:146-57.

6. Foeldvari I, Klotsche J, Kasapcopur O, Adrovic A, Stanevicha V, Sakmoto AP, et al. Update on the Juvenile Systemic Sclerosis Inception Cohort Project. Characteristics of the First 80 Patients at First Assessment. Arthritis Rheumatol 2016;68(Suppl 10):2391.

7. Kelsey CE, Torok KS. The Localized Scleroderma Cutaneous Assessment Tool: responsiveness to change in a pediatric clinical population. J Am Acad Dermatol 2013;69:214-20.

8. Foeldvari I, Wierk A. Healthy children have a significantly increased skin score assessed with the modified Rodnan skin score. Rheumatology (Oxford) 2006;45:76-8.

9. Scalapino K, Arkachaisri T, Lucas M, Fertig N, Helfrich DJ, Londino AV Jr, et al. Childhood onset systemic sclerosis: classification, clinical and serologic features, and survival in comparison with adult onset disease. J Rheumatol 2006;33:1004-13.

10. Martini G, Foeldvari I, Russo R, Cuttica R, Eberhard A, Ravelli A, et al. Systemic sclerosis in childhood: clinical and immunologic features of 153 patients in an international database. Arthritis Rheum 2006;54:3971-8.

11. Foeldvari I, Zhavania M, Birdi N, Cuttica RJ, de Oliveira $\mathrm{SH}$, Dent PB, et al. Favourable outcome in 135 children with juvenile systemic sclerosis: results of a multi-national survey. Rheumatology (Oxford) 2000;39:556-9.

12. Russo RA, Katsicas MM. Clinical characteristics of children with Juvenile Systemic Sclerosis: follow-up of 23 patients in a single tertiary center. Pediatr Rheumatol Online J 2007;5:6.

13. Misra R, Singh G, Aggarwal P, Aggarwal A. Juvenile onset systemic sclerosis: a single center experience of 23 cases from Asia. Clin Rheumatol 2007;26:1259-62.

14. Cutolo M, Sulli A, Pizzorni C, Accardo S. Nailfold videocapillaroscopy assessment of microvascular damage in systemic sclerosis. $J$ Rheumatol 2000;27:155-60.

15. Clements PJ, Lachenbruch PA, Seibold JR, Zee B, Steen VD, Brennan P, et al. Skin thickness score in systemic sclerosis: an assessment of interobserver variability in 3 independent studies. $\mathrm{J}$ Rheumatol 1993;20:1892-6.

16. La Torre F, Martini G, Russo R, Katsicas MM, Corona $F$, Calcagno $G$, et al. A preliminary disease severity score for juvenile systemic sclerosis. Arthritis Rheum 2012;64:4143-50.

17. Arkachaisri T, Vilaiyuk S, Torok KS, Medsger TA Jr. Development and initial validation of the localized scleroderma skin damage index and physician global assessment of disease damage: a proof-of-concept study. Rheumatology (Oxford) 2010;49:373-81.

18. Atzeni F, Bardoni A, Cutolo M, Hunzelmann N, Krieg T, Martini G, et al. Localized and systemic forms of scleroderma in adults and children. Clin Exp Rheumatol 2006;24:36-45.

19. Hedrich CM, Fiebig B, Hahn G, Suttorp M, Gahr M. Presentations and treatment of childhood scleroderma: localized scleroderma, eosinophilic fasciitis, systemic sclerosis, and graft-versus-host disease. Clin Pediatr (Phila) 2011;50:604-14.

20. Martini G, Vittadello F, Kasapçopur O, Magni Manzoni $\mathrm{S}$, Corona F, Duarte-Salazar C, et al. Factors affecting survival in juvenile systemic sclerosis. Rheumatology (Oxford) 2009;48:119-22.

21. Borowiec A, Dabrowski R, Wozniak J, Jasek S, Chwyczko T, Kowalik I, et al. Cardiovascular assessment of asymptomatic patients with juvenile-onset localized and systemic scleroderma: 10 years prospective observation. Scand J Rheumatol 2012;41:33-8.

22. Adrovic A, Oztunc F, Barut K, Koka A, Gojak R, Sahin $\mathrm{S}$, et al. The frequency of pulmonary hypertension in patients with juvenile scleroderma. Bosn J Basic Med Sci 2015;15:30-5. 
23. Postolova A, Chen JK, Chung L. Corticosteroids in Myositis and Scleroderma. Rheum Dis Clin North Am 2016;42:103-18.

24. Yilmaz N, Can M, Kocakaya D, Karakurt S, Yavuz S. Two-year experience with mycophenolate mofetil in patients with scleroderma lung disease: a case series. Int J Rheum Dis 2014;17:923-8.

25. Elhai M, Meunier M, Matucci-Cerinic M, Maurer B, Riemekasten G, Leturcq T, et al. Outcomes of patients with systemic sclerosis-associated polyarthritis and myopathy treated with tocilizumab or abatacept: a EUSTAR observational study. Ann Rheum Dis 2013;72:1217-20.

26. Martini G, Ramanan AV, Falcini F, Girschick H, Goldsmith DP, Zulian F. Successful treatment of severe or methotrexate-resistant juvenile localized scleroderma with mycophenolate mofetil. Rheumatology (Oxford) 2009;48:1410-3.

27. Li SC, Torok KS, Pope E, Dedeoglu F, Hong $\mathrm{S}$, Jacobe HT, et al. Development of consensus treatment plans for juvenile localized scleroderma: a roadmap toward comparative effectiveness studies in juvenile localized scleroderma. Arthritis Care Res (Hoboken) 2012;64:1175-85.
28. Rosenkranz ME, Agle LM, Efthimiou P, Lehman TJ. Systemic and localized scleroderma in children: current and future treatment options. Paediatr Drugs 2006;8:85-97.

29. Shimizu M, Hashida Y, Ueno K, Yokoyama T, Nakayama Y, Saito T, et al. Successful treatment with bosentan for pulmonary hypertension and reduced peripheral circulation in juvenile systemic sclerosis. Pediatr Cardiol 2011;32:1040-2.

30. Shima Y, Kuwahara Y, Murota H, Kitaba S, Kawai M, Hirano T, et al. The skin of patients with systemic sclerosis softened during the treatment with anti-IL-6 receptor antibody tocilizumab. Rheumatology (Oxford) 2010;49:2408-12.

31. Daoussis D, Melissaropoulos K, Sakellaropoulos G, Antonopoulos I, Markatseli TE, Simopoulou T, et al. A multicenter, open-label, comparative study of B-cell depletion therapy with Rituximab for systemic sclerosis-associated interstitial lung disease. Semin Arthritis Rheum 2017;46:625-31.

32. Fraticelli P, De Vita S, Franzolini N, Svegliati S, Scott $\mathrm{CA}$, Tonnini $\mathrm{C}$, et al. Reduced type I collagen gene expression by skin fibroblasts of patients with systemic sclerosis after one treatment course with rituximab. Clin Exp Rheumatol 2015;33:160-7. 